\title{
Differential Strategy Research for Vehicle with Motorized Wheels Based on Torque Control
}

\author{
Chuanxue Song ${ }^{\mathrm{a}}$, Silun Peng ${ }^{\mathrm{b}}$,Liqiang Jin, Shengyi Xuan and Zhu'an Zheng \\ Automotive College of Jilin University, Changchun 130025, China \\ asongchx@126.com, ${ }^{b}$ pengs111@mials.ju.edu.cn
}

\begin{abstract}
Keywords: Motorized Wheel; Differential Strategy; Torque Control; Neural Network.
\end{abstract}
Abstract. According to the electronic differential problems for vehicle with motorized wheels, the electronic differential control strategy based on torque control is designed through BP neural network theory, and a suitable BP network is trained with the help of Matlab toolbox. The consistency of both sides on wheel slip ratio is achieved through the differential controller. The controller contains two models. One is BP neural network model which calculates demand regulating by longitudinal speed and steering wheel angle. The other is electronic throttle distribution model which calculates the target opening degree of electronic throttle on two sides by torque regulating variable. The validity of control strategy is confirmed by simulation results, which show that the differential problem is well solved by the control strategy.

\section{Introduction}

With the rise of the electric vehicle, the vehicle with motorized wheels began to attract more and more attention. Compared with the traditional centralized driven vehicle, the motorized wheel driven has incomparable advantages. It is the car's future. This kind of driven adds the degrees of control freedom. So to ensure the reliable and efficient driving, the differential control should be solved as one of the key technologies.

The present research is mainly based on the Ackerman model to get the relations of each wheel. And the control strategy is built according to these relations. As the Ackerman model is an ideal simplified model, there are various methods to improve it [1]. However in the real driving, the road condition is very complicated, and the degrees of freedom are limited with the control strategy. Being unstable will often come about during the bigger steering angle and higher speed.

As for above shortages mentioned, Jilin University studied the electronic differential control strategies based on torque control and found that the problem caused by the different turning radius between inside and outside driven wheel is no longer prominent with the torque control [2]. Wuhan University of Technology studies the control strategy which distributes the drive torque according to the wheel load [3]. The slip rate can be reduced by this method. But as the result of vehicle non-linear characteristics, the effect is very limited. Ge-Yinghui and Zhao-yan'e choose the slip rate as the control target $[4,5]$ and get a very good result through simulation. But the slip rate is hard to get very precisely in real vehicle. By the above analysis, we can see that the electronic differential technology has not been effectively resolved.

In view of the nonlinear system of vehicle and difficult to obtain real-time feedback of slip rate mentioned above, this paper designs the control strategy according to BP neural network theory.

\section{Control Strategy Design}

System Structure. When the vehicle is turning, one side tire slip rate is higher than the other side. It is essentially caused by centrifugal force, which is decided by vehicle speed and steering angle. So the longitudinal speed $(\mathrm{u})$, steering wheel angle $\left(\delta_{\mathrm{sw}}\right)$ and opening degree of throttle $\left(\alpha_{\mathrm{p}}\right)$ are adopted as the controller inputs. The demand torque is generally the input of motor controller. Thus the electronic throttle means the demand torque. And the distribution of motor torque can be achieved by distributing opening degree of electronic throttle of two sides. In this paper, the electronic throttles of two sides are 
defined as $\alpha_{\mathrm{ml}}$ and $\alpha_{\mathrm{mr}}$, which are the outputs of controller. The whole control system structure are shown as Fig.1.

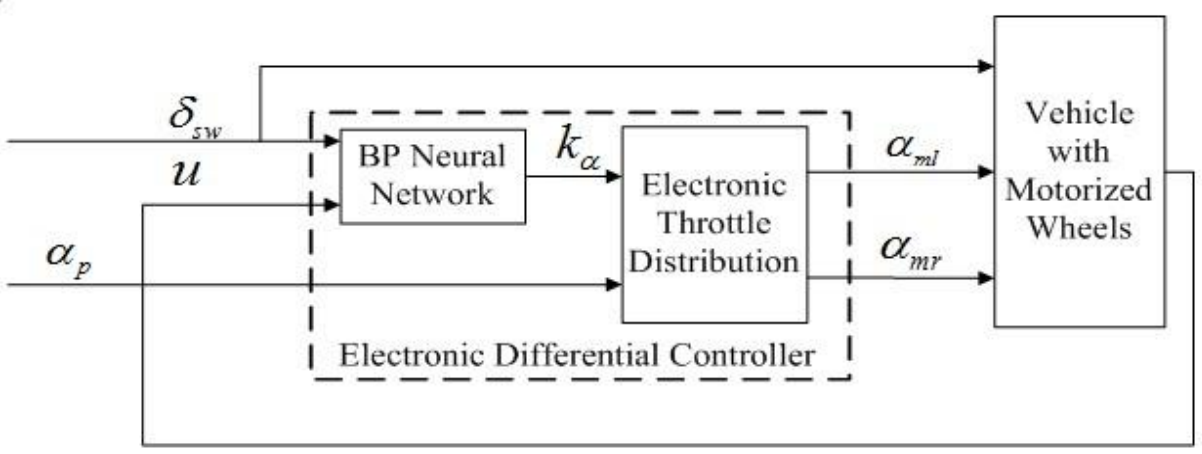

Fig.1 Control system structure

The electronic differential controller contains two models. One is BP neural network model, which calculates demand regulating by longitudinal speed and steering wheel angle. Hence, the inputs of the model are speed and angle, and the output is the distribution percentage of electronic throttle on two sides $\left(\mathrm{k}_{\alpha}\right)$, which is called torque regulating variable. The other is electronic throttle distribution model, which calculates the target opening degree of electronic throttle on two sides by torque regulating variable. The calculating is according to Eq. 1.

$\left\{\begin{array}{l}\alpha_{m l}=\alpha_{p}\left(1-k_{\alpha}\right) \\ \alpha_{m r}=\alpha_{p}\left(1+k_{\alpha}\right)\end{array}\right.$

When the vehicle is driving in high speed or urgent acceleration, the driven ability of motorized wheels may be close to its limitation. If the vehicle is turning at this time, according to Eq. 1, the dynamic property must be getting worse. So the limit that the whole driving force mustn't be changed should be added in the electronic throttle distribution model.

Control Strategy Design. The input and output layer structure of BP neural network can be deduced by control system structure. The input layer includes two nodes which represent vehicle speed and steering wheel angle input. The output layer includes one node which represents torque regulating variable.

In order to initially verify the feasibility of this method, about 700 simulation data are got from the simulation model introduced by reference [7], which is a 9 degrees of freedom model for electric vehicle with motorized Wheels. The data are the best torque regulating amount in different situations which the vehicle speed is from 0 to $135 \mathrm{~km} / \mathrm{h}$ and the steering wheel angle is from 0 to 640 degrees. The BP neural network is trained base on these data samples. Among them, 40 data samples are chose to verify the network performance.

In order to facilitate controller design, the Matlab toolbox of neural network is used for aided design. For different network model, the Matlab toolbox supplies various learning algorithm and related toolbox function. The neural network can be visualized and conveniently designed and calculated.

After repeated training, a $2 \times 12 \times 1 \mathrm{BP}$ network structure is finally determined. Its training parameters are shown in the Table 1.

Table 1 BP network training parameters

\begin{tabular}{l|l}
\hline Learning Algorithm & Trainlm \\
\hline $\begin{array}{l}\text { Transition Function of } \\
\text { Hidden Layer Nodes }\end{array}$ & Tansig \\
\hline $\begin{array}{l}\text { Transition Function of } \\
\text { Output Layer Nodes }\end{array}$ & Purelin \\
\hline Iterations & 1000 \\
\hline Learning Rate & 0.01 \\
\hline
\end{tabular}


\begin{tabular}{l|l}
\hline Learning Target & e-6 \\
\hline
\end{tabular}

In order to test the network performance, 40 data samples are predicted through BP network which designed above. The percentage errors of prediction are shown in Fig. 2.

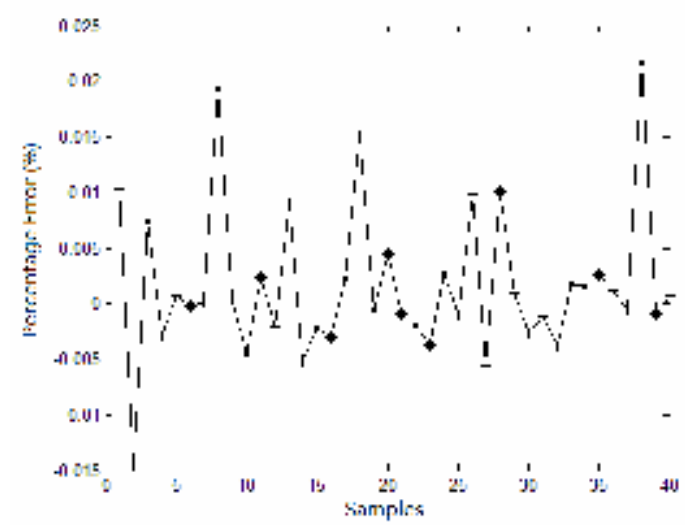

Fig. 2 Percentage error

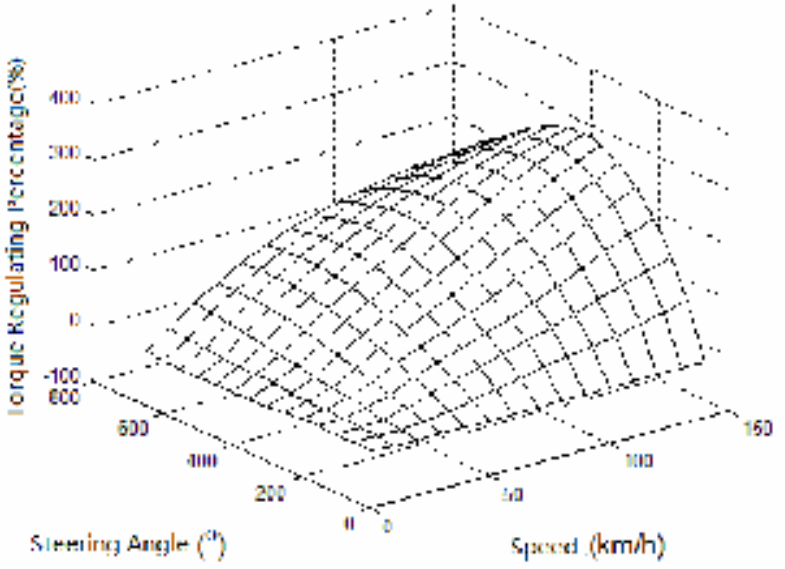

Fig. 3 Input-output MAP

As can be seen from Fig. 2, the forecast errors for new samples of the designed network are very low. So this network has a high accuracy prediction and good generalization ability. The input-output MAP of BP neural network is shown in Fig. 3.

Control Strategy Simulation. The designed electronic differential controller is integrated into the simulation model introduced by reference [7], to test the performance of the control strategy.

The vehicle is accelerated for $15 \mathrm{~s}$ to reach $60 \mathrm{~km} / \mathrm{h}$. Then a $40^{\circ}$ angle step input is added to the steering wheel. The slip rate curves during equal torque distribution control and electronic differential control are shown in Fig. 4.

As can be seen from Fig. 4, while the driven torque is distributed equally, the slip rate is significant difference between two sides. But after use electronic differential controller, the slip rate of outside motorized wheel is very close to inside and the whole slip rate level is getting down. Through many times simulation, it has been found that the slip rate will get down about $1 \%$ at high speed and big steering angle. It is very effective.

In order to test controller performance during different steering angle and speed, the vehicle is kept accelerating from $20 \mathrm{~km} / \mathrm{h}$ for $50 \mathrm{~s}$. During the accelerating, the accelerator pedal is keeping $60 \%$ opening and the steering wheel is added a sine signal which amplitude is $60^{\circ}$. In the end of the simulation, the final speed is about $85 \mathrm{~km} / \mathrm{h}$. the slip rate curves of two sides are shown in Fig. 5 .

From Fig. 5, it can be seen that the slip rate will increase along with the increase of vehicle speed. But the consistent of slip rate on two sides can be guaranteed by electronic differential controller. During the simulation, the slip rates are changed along with steering angle and vehicle speed. But the slip rates of two sides are always kept consistent. Thus the controller has a good performance at commonly used range of vehicle speed and steering angle.

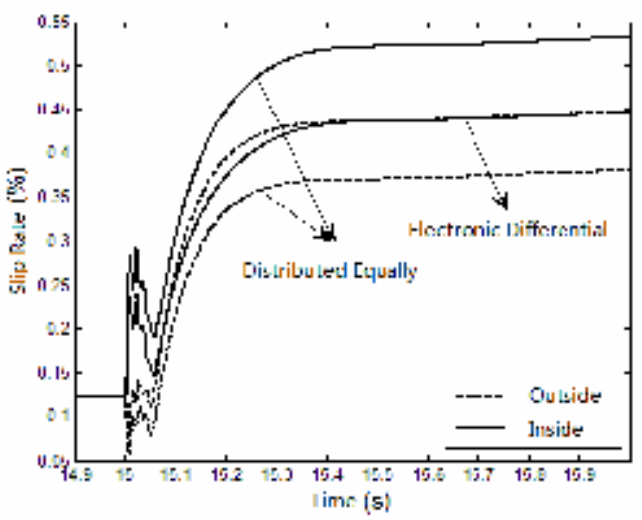

Fig. 4 Slip response contrast

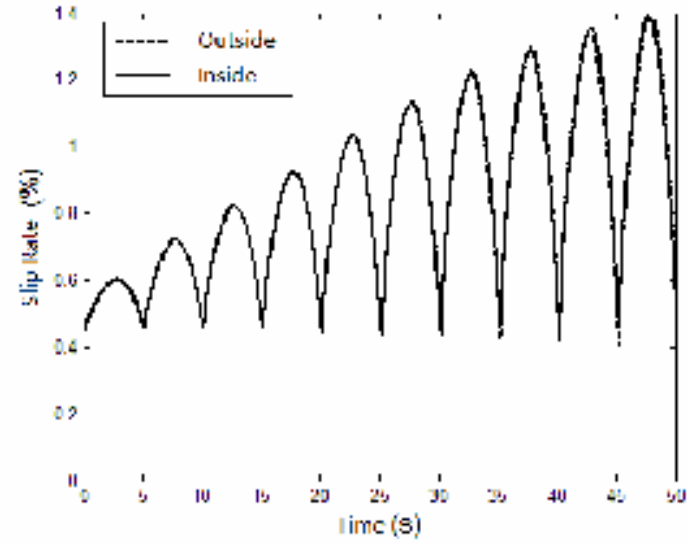

Fig. 5 Slip curve on both sides 


\section{Conclusion}

In the light of the electronic differential problems for vehicle with motorized wheels, the BP neural network theory is used to design control strategy. With the help of Matlab toolbox, a suitable BP network is trained. The electronic differential control strategy is verified through simulation. And the results show that the controller can keep consistence of two sides slip rate under commonly range of speed and steering angle, which means the differential problem is well solved by the control strategy.

\section{References}

[1] Gair S, Cruden A, McDonald J and Hredzak B: IEEE International Conference (Mechatronics, June 3-5, 2004). Vol. 1, p. 98-103.

[2] Jin Liqiang, Wang Qingnian, Zhou Xuehu, and Song Chuanxue: Journal of Jilin University Engineering and Technology Edition, Vol.38 (2008) No.2, p.1-6.

[3] Wang Kang: Research of In-wheel Drive Control System in Electric Vehicle's Technology. (MS., Wuhan University of Technology, China 1998), p.71

[4] Ge Yinghui, Li Guangzheng: Journal of Zhejiang University (Engineering Science), Vol.39 (2005) No.12, p.1973-1978.

[5] Zhao Yan'e, Zhang Jianwu: Journal of System Simulation, Vol.20 (2008) No.18, p.4767-4771+4775.

[6] Bose BK: IEEE International Conference on Industrial Technology (Dec 14-17, 2005). Vol.1, p. 20-64.

[7] Si-lun P, Yun-Bing Y. 2nd International Conference on Energy and Environment Technology, (Changsha, Dec 5-7, 2010). Vol.2, p. 415-418. 